APS/123-QED

\title{
Suppression of Higgsino mediated proton decay by cancellations in GUTs and strings
}

\author{
Pran Nath* and Raza M. Syed \\ Department of Physics, Northeastern University, Boston, MA 02115-5000, USA.
}

(Dated: October 22, 2018)

\begin{abstract}
A mechanism for the enhancement for proton lifetime in supersymmetric/supergravity (SUSY/SUGRA) grand unified theories (GUTs) and in string theory models is discussed where Higgsino mediated proton decay arising from color triplets (anti-triplets) with charges $Q=-1 / 3(1 / 3)$ and $Q=-4 / 3(4 / 3)$ is suppressed by an internal cancellation due to contributions from different sources. We exhibit the mechanism for an $S U(5)$ model with $45_{H}+\overline{45}_{H}$ Higgs multiplets in addition to the usual Higgs structure of the minimal model. This model contains both $Q=-1 / 3(1 / 3)$ and $Q=-4 / 3(4 / 3)$ Higgs color triplets (anti-triplets) and simple constraints allow for a complete suppression of Higgsino mediated proton decay. Suppression of proton decay in an $S U(5)$ model with Planck scale contributions is also considered. The suppression mechanism is then exhibited for an $S O(10)$ model with a unified Higgs structure involving $144_{H}+\overline{144}_{H}$ representations. The $S U(5)$ decomposition of $144_{H}+\overline{144}_{H}$ contains $5_{H}+\overline{5}_{H}$ and $45_{H}+\overline{45}_{H}$ and the cancellation mechanism arises among these contributions which mirrror the $S U(5)$ case. The cancellation mechanism appears to be more generally valid for a larger class of unification models. Specifically the cancellation mechanism may play a role in string model constructions to suppress proton decay from dimension five operators. The mechanism allows for the suppression of proton decay consistent with current data allowing for the possibility that proton decay may be visible in the next round of nucleon stability experiment.
\end{abstract}

PACS numbers: 12.10.Dm; 13.30.- a; 12.10.- g; 11.25.Wx; 11.30.Pb; 12.60.- i

*Electronic address: nath@lepton.neu.edu

${ }^{\dagger}$ Electronic address: r.syed@neu.edu 


\section{INTRODUCTION}

Grand unification and strings are attractive schemes for the unification of interactions. One consequence of grand unification is that one has baryon and lepton number non-conservation which can lead to proton decay 1, 2, 3] and a similar phenomenon occurs in string models (for a review see [4]). In supersymmetric and supergravity GUT theories[5, 6] baryon and lepton number violating dimension five operators are the dominant contributions [7, 8]. These contributions are now stringently constrained by experiment. Thus the analysis of Ref.[9] indicates that the minimal $S U(5)$ even in the decoupling limit is eliminated [9] (we note in passing that the minimal $S U(5)$ model is eliminated in any case since it fails to reproduce the fermion masses) and further that supersymmetric grand unification in general may also be under siege[10] due to the current experimental lower limits on the proton lifetime[11, 12]. While there are ways to lift the siege (see, e.g., [13]) the proton lifetime limit is certainly one of the most important constraints on grand unification and on string models, and is likely to become even more stringent as the error corridor on the predictions decrease and the lower limits from experiment improve. Several possible avenues for suppressing proton decay have already been discussed in the literature from mild suppression using textures[14], and CP phases[15] to stronger suppression[16], and suppression up to the current limit of experiment [17]. Here we add to this list the cancellation mechanism for the suppression of proton decay from dimension five operators which is inspired by a similar mechanism used to suppress the EDM of the electron and of the neutron in supersymmetric theories[18].

The outline of the rest of the paper is as follows: in Sec. II we discuss the constraints necessary for the suppression of baryon and lepton number violating dimension five operators arising from Higgsino exchange. These constraints are valid both for grand unified theories as well as for models arising from strings. In Sec. III we discuss an $S U(5)$ grand unification model where we include a $45+\overline{45}$ plet of Higgs in addition to the usual Higgs structure of the minimal $S U(5)$. We show that a suppression of the dimension five operators can be achieved in this case via a cancellation between contributions from the $5_{H}+\overline{5}_{H}$ and from the $45_{H}+\overline{45}_{H}$. In Sec. IV we discuss the cancellation mechanism for an $S U(5)$ model with Planck scale contributions. In Sec. V, we extend this analysis to $S O(10)$ GUT, where we consider the recently proposed model based on a unified Higgs sector. Specifically, we consider the model 
where the Higgs sector consists of the $\mathrm{SO}(10)$ irreducible representations $144_{H}+\overline{144}_{H}$, which allow one to break the $S O(10)$ gauge group all the way down to $S U(3)_{C} \times U(1)_{e m}$. The $S U(5)$ decomposition of $144_{H}\left(\overline{144}_{H}\right)$ contains $\overline{45}_{H}\left(45_{H}\right)$ of $S U(5)$ Higgs representations. Thus in this case a mechanism similar to that of Sec. III for the cancellation of baryon and lepton number violating dimension five operators can also be implemented. While the analyses in Secs. III- $\mathrm{V}$ are for specific models, the cancellation mechanism for the suppression of baryon and lepton number violating dimension five operators may be more general and applicable to a larger class of models. Conclusions are given in Sec. VI.

\section{SUPPRESSION OF HIGGSINO MEDIATED PROTON DECAY IN GUTS AND STRINGS}

In this section we consider the constraints that are necessary for the suppression or complete elimination of all baryon and lepton number violating dimension five operators in grand unified or in string theory models. Thus in such models below the unification scale for grand unified theory or below the string scale for string theory the Standard Model gauge group invariance, i.e., invariance under $S U(3)_{C} \times S U(2) \times U(1)_{Y}$ prevails. For the sake of the analysis below we assume that the doublet-triplet problem is resolved with one pair of Higgs doublets light and all the remaining Higgs doublets and all the Higgs triplets are heavy. Now grand unified theories and string theories in general will generate Higgs triplets (anti-triplets) with charges $Q=-1 / 3(1 / 3)$ and $Q=-4 / 3(4 / 3)$. We denote the Higgs triplets (anti-triplets) with charges $Q=-1 / 3(1 / 3)$ by $H_{q}^{\alpha}\left(H_{q \alpha}^{\prime}\right)(q=1,2, . ., n)$ and $\alpha=1,2,3$ is the color index, and Higgs triplets (anti-triplets) with charges $Q=-4 / 3(4 / 3)$ by $\tilde{H}_{q^{\prime}}^{\alpha}\left(\tilde{H}_{q^{\prime} \alpha}^{\prime}\right)\left(q^{\prime}=1,2, . ., m\right)$. The $S U(3)_{C} \times S U(2)_{L} \times U(1)_{Y}$ invariant superpotential below the unification scale may then be written as

$$
\begin{array}{r}
\left(H_{q \alpha}^{\prime} \mathcal{M}_{q p} H_{p}^{\alpha}+J_{q \alpha} H_{q}^{\alpha}+H_{q \alpha}^{\prime} K_{q}^{\alpha}\right) \\
+\left(\tilde{H}_{q^{\prime} \alpha}^{\prime} \tilde{\mathcal{M}}_{q^{\prime} p^{\prime}} \tilde{H}_{p^{\prime}}^{\alpha}+\tilde{J}_{q^{\prime} \alpha} \tilde{H}_{q^{\prime}}^{\alpha}+\tilde{H}_{q^{\prime} \alpha}^{\prime} \tilde{K}_{q^{\prime}}^{\alpha}\right) .
\end{array}
$$

For the matter content of MSSM, with three generations of quarks and leptons, the sources $J$ and $K$ have the following form

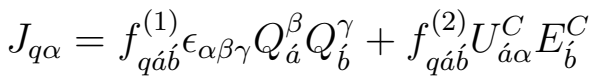

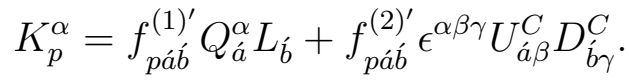


Here $Q_{\dot{a}}\left(L_{\dot{b}}\right)$ are quark(lepton) $S U(2)_{L}$ doublets, and $U_{a}^{C}, D_{\hat{b}}^{C}\left(E_{a}^{C}\right)$ are $S U(2)_{L}$ singlets, where $\dot{a}, \hat{b}=1,2,3$ are the generation indices. For the tilde sources $\tilde{J}$ and $\tilde{K}$ one has the form

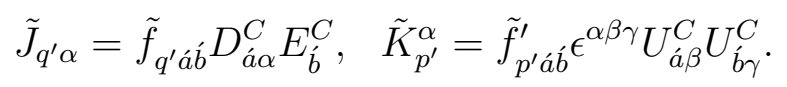

Now suppose we make unitary transformation and go to a basis where only $H_{1}$ and $H_{1}^{\prime}$ (in the new basis) couple with the matter fields. Then it is easily seen that the condition that kills the baryon and lepton number violating dimension five operators of $L L L L$ type is as follows

$$
\left(U_{a \dot{a} b}^{(1)} \mathcal{M} V_{\dot{c} d}^{(1) T}\right)_{11}^{-1}+\Lambda_{a \dot{a} b \dot{c} \dot{d}}^{Q G}=0
$$

while for the suppression of baryon and lepton number violating interactions of type RRRR one has the constraint

$$
\left(U_{a \dot{a} b}^{(2)} \mathcal{M} V_{\dot{c} \dot{d}}^{(2) T}\right)_{11}^{-1}+\left(\tilde{U}_{\dot{a} \dot{b}} \tilde{\mathcal{M}} \tilde{V}_{\dot{c} d}^{T}\right)_{11}^{-1}+\tilde{\Lambda}_{a \dot{a} \dot{b} \dot{d}}^{Q G}=0
$$

Here $U$ and $V$, and $\tilde{U}$ and $\tilde{V}$ are unitary matrices that take us to the basis where only $H_{1}$ and $H_{1}^{\prime}$ couple with matter. We note that the matrices $\mathcal{M}$ and $\tilde{\mathcal{M}}$ as well as the sources $J, K, \tilde{J}, \tilde{K}$ may contain Planck scale contributions as exhibited explicitly in Sec.(IV). However, in addition one may have quantum gravity $(\mathrm{QG})$ corrections which we have exhibited by $\Lambda^{Q G}$ and $\tilde{\Lambda}^{Q G}$ terms in Eqs. (4) and (5) to take account of such effects. In this work we do not consider the quantum gravity corrections although such corrections could also be utilized for the suppression of B\&L violating dimension five operators6 in grand unified and string theory models. If we are already in the basis where only $H_{1}$ and $H_{1}^{\prime}$ couple with matter, then $U^{(1)}=I=V^{(1)}, U^{(2)}=I=V^{(2)}$, and $\tilde{U}=I=\tilde{V}$, and if we ignore the quantum gravity effects then one gets the familiar condition[19] $\mathcal{M}_{11}^{-1}=0$ when only the Higgs triplets (anti-triplets) with charges $Q=-1 / 3(1 / 3)$ are considered in the analysis. The constraints of Eqs.(4) and (5) together are then sufficient to kill all baryon and lepton number violating dimension five operators in any grand unified theory or in any string theory model arising from Higgsino exchange. The constraints of Eqs.(4) and (5) are very stringent because of their dependence on generation indices. However, significant simplification will occur in specific unified models. Below we discuss two models, one in $\mathrm{SU}(5)$ and the other in $\mathrm{SO}(10)$ where the constraints of Eqs.(44) and (5) can be satisfied by internal cancellations. In 
the analysis below we consider cases where proton decay is suppressed via the cancellation mechanism both in the absence of the Planck scale contributions (Sec.III) as well as when Planck scale contributions are taken into account (Secs IV and V).

\section{THE CANCELLATION MECHANISM IN SU(5) GRAND UNIFICATION}

In this section we illustrate the satisfaction of Eqs.(44) and (5) in the context of an $S U(5)$ model. The Higgs sector of the minimal $S U(5)$ model consists of a $24_{H}$ of Higgs to break the GUT symmetry and a pair of $5_{H}+\overline{5}_{H}$ to break the electro-weak symmetry. Typically in this model a fine tuning is needed to obtain the doublet-triplet splitting. We expand now the Higgs sector by inclusion of a pair of $45_{H}+\overline{45}_{H}$ of Higgs (for the use of 45 plet in $S U(5)$ model building see [20]). In this case the superpotential for the Yukawa couplings is of the form

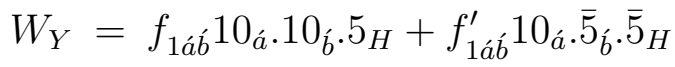

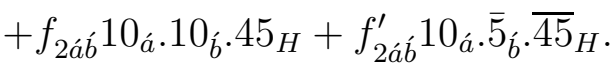

For the Higgs superpotential we choose

$$
\begin{aligned}
W_{H}= & M_{5} \overline{5}_{H} 5_{H}+h_{1} \overline{5}_{H} \cdot 24_{H} \cdot 5_{H}+h_{2} \overline{5}_{H} \cdot 24_{H} \cdot 45_{H}+h_{3} 5_{H} \cdot 24_{H} \cdot \overline{45}_{H} \\
& +h_{4} \bar{M} 45_{H} \cdot \overline{45}_{H}+h^{\prime} W_{H}^{\prime}\left(24_{H}\right) .
\end{aligned}
$$

Here $W_{H}^{\prime}\left(24_{H}\right)$ generates spontaneous breaking producing a VEV of the form

$$
<24_{H}>=\operatorname{diag}(2,2,2,-3,-3) M,
$$

and breaks $S U(5) \rightarrow S U(3) \times S U(2) \times U(1)_{Y}$ and we assume that there is no VEV growth for the 45 plet of Higgs. In the above we adopt the fine tuning that is conventionally used to produce a light Higgs . We exhibit this explicitly. The Higgs doublets arise from both $5_{H}+\overline{5}_{H}$ and from $45+\overline{45}_{H}$ and we denote these by $H^{a}\left(5_{H}\right), P^{a}\left(45_{H}\right)$, and by $H_{a}^{\prime}\left(\overline{5}_{H}\right)$, $Q_{a}\left(\overline{45}_{H}\right)$. The mass matrix is given by

$$
\begin{aligned}
& H^{a} \quad P^{a} \\
& \begin{array}{l}
H_{a}^{\prime} \\
Q_{a}
\end{array}\left(\begin{array}{ll}
M_{11}^{d} & M_{12}^{d} \\
M_{21}^{d} & M_{22}^{d}
\end{array}\right),
\end{aligned}
$$


where for the model of Eq.(17)

$$
\begin{aligned}
M_{11}^{d}=-3 M h_{1}+M_{5}, \quad M_{12}^{d} & =-\frac{5 \sqrt{3}}{2 \sqrt{2}} M h_{2}, \quad M_{21}^{d}=-\frac{5 \sqrt{3}}{2 \sqrt{2}} M h_{3}, \\
M_{22}^{d} & =\bar{M} h_{4} .
\end{aligned}
$$

We denote the Higgs triplets by $H^{\alpha}\left(5_{\overline{144}}\right), Q^{\alpha}\left(5_{144}\right), P^{\alpha}\left(45_{\overline{144}}\right)$ and the anti-triplets by $H_{\alpha}^{\prime}\left(\overline{5}_{144}\right), Q_{\alpha}\left(\overline{45}_{144}\right), P_{\alpha}\left(\overline{5}_{\overline{144}}\right)$. Here $H^{\alpha}, Q^{\alpha}\left(H_{\alpha}^{\prime}, Q_{\alpha}\right)$ have charges $-1 / 3(1 / 3)$ while $Q^{\alpha}$ $\left(P_{\alpha}\right)$ have charges -4/3(4/3). In the basis where the columns are $H^{\alpha}, P^{\alpha}, Q^{\alpha}$ and the rows are $H_{\alpha}^{\prime}, Q_{\alpha}, P_{\alpha}$, the Higgs triplet mass matrix has the following form

$$
\begin{aligned}
& H^{\alpha} \quad P^{\alpha} \quad Q^{\alpha} \\
& \begin{array}{l}
H_{\alpha}^{\prime} \\
Q_{\alpha} \\
P_{\alpha}
\end{array}\left(\begin{array}{ccc}
M_{11} & M_{12} & 0 \\
M_{21} & M_{22} & 0 \\
0 & 0 & M_{33}
\end{array}\right),
\end{aligned}
$$

where for the model of Eq.(7)

$$
\begin{gathered}
M_{11}=2 M h_{1}+M_{5}, \quad M_{12}=-\frac{5}{\sqrt{2}} M h_{2}, \quad M_{21}=-\frac{5}{\sqrt{2}} M h_{3}, \\
M_{22}=\bar{M} h_{4}, \quad M_{33}=\bar{M} h_{4} .
\end{gathered}
$$

From Eq.(91) one finds that all the doublets are heavy and one needs a fine tuning to get a light Higgs doublet. This fine tuning condition is

$$
h_{4}=-\frac{25 M}{4 \bar{M}} h_{2} h_{3}\left(h_{1}-\frac{M_{5}}{3 M}\right)^{-1}
$$

With this constraint the second pair of Higgs doublets are heavy and do not participate in low energy physics. Further, with the constraint of Eq.(13) all the three Higgs triplets given by Eq.(11) are heavy.

The Higgs triplet interactions are

$$
\begin{aligned}
W_{i n t}= & \left(J_{1 \alpha} H^{\alpha}+J_{2 \alpha} P^{\alpha}+H_{\alpha}^{\prime} K_{1}^{\alpha}+Q_{\alpha} K_{2}^{\alpha^{\prime}}\right) \\
& +\left(\tilde{J}_{\alpha} Q^{\alpha}+\tilde{K}^{\alpha} P_{\alpha}\right),
\end{aligned}
$$

where $J_{1}^{\alpha}, K_{1 \alpha}$ etc. are the matter currents to which the color Higgs fields couple. We assume that $J_{1}, K_{1}$ arise from $5_{H}+\overline{5}_{H}$ Higgs couplings, while the $J_{2}, K_{2}$ and $\tilde{J}, \tilde{K}$ arise 
from $45_{H}+\overline{45}_{H}$. In order to satisfy the constraint of Eq.(4) and Eq.(15) we make specific assumptions regarding the generational dependence of $45_{H}$ Higgs couplings relative to the $5_{H}$ Higgs couplings, and of $\overline{45}_{H}$ Higgs coupling relative to the $\overline{5}_{H}$ Higgs couplings as follows

$$
f_{2 a ́ b}=\lambda f_{1 a ́ b}, \quad f_{2 a ́ b}^{\prime}=\lambda^{\prime} f_{1 a ́ b}^{\prime} .
$$

In this case in the analysis of baryon and lepton number violating dimension five operators the generational dependence factors out and the entire left hand side of Eq.(44) and Eq.(5) is proportional to $f_{1 a ́ b} f_{1 c ́ d}^{\prime}$. On eliminating the Higgs triplets fields one finds lepton and baryon number violating operator of chirality $L L L L$ and of chirality $R R R R$. The $L L L L$ operators

arise from the elimination of the heavy Higgs fields $H_{\alpha}^{\prime}, Q_{\alpha} ; H^{\alpha}, P^{\alpha}$ with charges $\pm \frac{1}{3}$. The cancellation condition in this case is

$$
\sum_{i=1}^{4} C_{i}^{L}\left(\lambda, \lambda^{\prime}\right) h_{i}^{\prime}=0
$$

Here $h_{1}^{\prime}=\left(h_{1}+M_{5} / 2 M\right), h_{i}^{\prime}=h_{i}(\mathrm{i}=2,3,4)$, and $C_{i}^{L}\left(\lambda, \lambda^{\prime}\right)$ is a polynomial of the type $\left(a_{L}+b_{L} \lambda+c_{L} \lambda^{\prime}+d_{L} \lambda \lambda^{\prime}\right)$ where $a_{L}$ etc are numerical co-efficients. For the $B \& L$ violating dimension five operators of chirality $R R R R$, one finds that the contributions to them arise from the elimination of the heavy Higgs fields $H_{\alpha}^{\prime}, Q_{\alpha} ; H^{\alpha}, P^{\alpha}$ as well as from the elimination of the Higgs tripelts $P_{a}, Q^{a}$ with charges $\pm \frac{4}{3}$. The cancellation condition in this case is

$$
\sum_{i=1}^{4} C_{i}^{R}\left(\lambda, \lambda^{\prime}\right) \frac{\bar{M}}{M} h_{4} h_{i}^{\prime}-\lambda \lambda^{\prime} \operatorname{det}(\mathcal{H})=0
$$

where $\operatorname{det}(\mathcal{H})=\left(2 \frac{\bar{M}}{M} h_{1}^{\prime} h_{4}-\frac{25}{2} h_{2} h_{3}\right)$ and where $C_{i}^{R}$ are defined analogous to $C_{i}^{L}$. Eqs (13),(16),(17) constitute three constraints on four Higgs couplings $h_{i}(\mathrm{i}=1-4)$ and thus can be satisfied leaving one parameter still arbitrary. Specifically, there are no constraints aside from the parallelity condition of Eq.(15) on the matter couplings of the Higgs which can thus be used to fix the textures.

\section{THE CANCELLATION MECHANISM IN AN $S U(5)$ MODEL WITH PLANCK SCALE CONTRIBUTIONS}

In Secs (III) we have given an explicit demonstration of an $S U(5)$ model where the cancellation mechanism leads to a suppression of proton decay. The analysis of Sec.III, 
however, did not have any Planck scale contributions. In this section we give a further example of the cancellation mechanism in the context of an $S U(5)$ model including Planck scale contributions. Such Planck scale contributions are used to generate the hierachical stuctures for the quark-lepton textures. Thus in the notation of Sec.III we may write the effective superpotential at the GUT scale including Planck scale corrections in the form

$$
W=\sum_{n}\left(f_{1 n} 5.10 .5_{H} \frac{\Sigma^{n}}{M_{P l}^{n}}+f_{2 n} 10.10 .5_{H} \frac{\Sigma^{n}}{M_{P l}^{n}}\right)
$$

where the couplings are written in a schematic form. After spontaneous breaking $\Sigma$ develops a VEV of size $M$ (see Eq. (8) ) and the above contribute terms suppressed by powers of $\left(M / M_{P l}\right)^{n}$. An analysis of the quark-lepton textures using expansions up to $\left(\Sigma / M_{P l}\right)^{3}$ was carried out in [14]. The above analysis has been extended recently to include expansions up to $\left(\Sigma / M_{P l}\right)^{4}[21]$. The co-efficients $a_{5 L}\left(a_{5 R}\right)$ of the effective B\&L violating dimension 5 operators $L L L L(R R R R)$ take the form

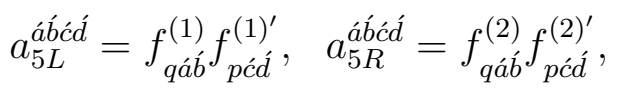

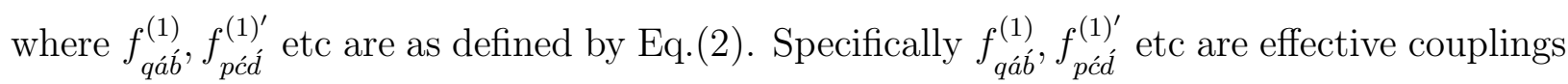
which are expansions in the Planck scale contributions. Their forms are explicitly exhibited in [21]. With the larger number of couplings available it is then possible to satisfy all the quark lepton textures. Further, one finds that solutions allow for the possibility that[21] $f_{p c ́ d}^{(1)^{\prime}}=0=f_{p c ́ d}^{(2)^{\prime}}$ which completely suppress the B\&L violating dimension five operators. This is an example of the cancellation mechanism where Planck scale corrections allow for the suppression of proton decay.

\section{SUPPRESSION OF BARYON AND LEPTON NUMBER VIOLATING DI- MENSION FIVE OPERATORS IN AN SO(10) MODEL}

In the $S U(5)$ model of Sec.III we saw that if there are more than one pair of Higgs triplets contributing to the generation of baryon and lepton number violating interactions, then there is the possibility of a partial or complete cancellation of these operators. In Sec,IV we saw the phenomenon of cancellation an $S U(5)$ model with Planck scale contributions . We consider now the $S O(10)$ case. There is already a considerable literature on model building in $\mathrm{SO}(10)$ (for some recent works see[17, 22, 23]). Here we will consider the $S O(10)$ model proposed 
recently with one step breaking down to the Standard Model gauge group and further down to the residual gauge group $S U(3)_{C} \times U(1)_{Y}$ using $144_{H}+\overline{14}_{H}$ of Higgs [24]. This case combines some features of the models discussed in Sec.III and in Sec.IV. Thus the model has more than one pair of Higgs triplets, and further, it has Planck scale contributions . Thus the quark-lepton masses for the first two generations (see Sec( $(\mathrm{VC})$ ) arise from the Planck scale contributions, while that of the third generation arise from the cubic interactions (see Eq.(43) ). We will compute the lepton and baryon number violating interactions for this model and show that a complete suppression of baryon and lepton number violating dimension 5 operators can occur in this case.

We begin by exhibiting the decomposition of 144 under $S U(5) \times U(1)$. Here one finds

$$
\begin{aligned}
144= & 5\left(\mathbf{Q}^{i}\right)[3]+\overline{5}\left(\mathbf{Q}_{i}\right)[7]+10\left(\mathbf{Q}^{i j}\right)[-1]+15\left(\mathbf{Q}_{(S)}^{i j}\right)[-1] \\
& +24\left(\mathbf{Q}_{j}^{i}\right)[-5]+40\left(\mathbf{Q}_{l}^{i j k}\right)[-1]+\overline{45}\left(\mathbf{Q}_{j k}^{i}\right)[3]
\end{aligned}
$$

where $i, j, k$ are the $\mathrm{SU}(5)$ indices and a similar decomposition of $\overline{144}$ holds so that we have

$$
\begin{aligned}
\overline{144}= & \overline{5}\left(\mathbf{P}_{i}\right)[-3]+5\left(\mathbf{P}^{i}\right)[-7]+\overline{10}\left(\mathbf{P}_{i j}\right)[1]+\overline{15}\left(\mathbf{P}_{i j}^{(S)}\right)[1] \\
& +24\left(\mathbf{P}_{j}^{i}\right)[5]+\overline{40}\left(\mathbf{P}_{i j k}^{l}\right)[1]+45\left(\mathbf{P}_{k}^{i j}\right)[-3]
\end{aligned}
$$

We note that the decomposition of $144_{H}+\overline{144}_{H}$ contains $5+\overline{5}$ pairs of Higgs, as well as a pair of $45_{H}+\overline{45}_{H}$ of Higgs. Thus in this sense it contains the essential ingredients of the $S U(5)$ model which has $5+\overline{5}$ and $45_{H}+\overline{45}_{H}$ of Higgs fields. There is then a good chance that a cancellation mechanism works in this case as well. We will show later in this section that this is indeed the case. The analysis in the rest of this section is as follows: in Sec. (VA) we give a brief discussion of spontaneous breaking with $144_{H}+\overline{144}_{H}$ of Higgs. In Sec. (VB) we discuss the doublet -triplet splitting. In Sec. (VC) we give an $S U(5) \times U(1)$ decomposition of couplings of matter and Higgs. Here we analyze quartic interactions as well as cubic interactions[25] when additional 10 and 45 of matter are introduced in order to generate large masses for the third generation of quarks and leptons. An analysis of baryon and lepton number violating interactions is given in Sec. (VD) where the condition for the complete suppression of baryon and lepton number violating LLLL and RRRR operators by the cancellation mechanism is discussed. 


\section{A. Spontaneous symmetry breaking}

To discuss the spontaneous breaking with $144_{H}+\overline{144}_{H}$ of Higgs, we consider the following form for the superpotential

$$
\begin{aligned}
\mathrm{W}= & M\left(\overline{144}_{H} \times 144_{H}\right) \\
& +\frac{\lambda_{45_{1}}}{M^{\prime}}\left(\overline{144}_{H} \times 144_{H}\right)_{45_{1}}\left(\overline{144}_{H} \times 144_{H}\right)_{45_{1}} \\
& +\frac{\lambda_{45_{2}}}{M^{\prime}}\left(\overline{144}_{H} \times 144_{H}\right)_{45_{2}}\left(\overline{144}_{H} \times 144_{H}\right)_{45_{2}} \\
& +\frac{\lambda_{210}}{M^{\prime}}\left(\overline{144}_{H} \times 144_{H}\right)_{210}\left(\overline{144}_{H} \times 144_{H}\right)_{210} .
\end{aligned}
$$

In the above the $45_{1}, 45_{2}$ and 210 couplings are defined as follows

$$
\begin{array}{r}
\left(\overline{144}_{H} \times 144_{H}\right)_{45_{1}}\left(\overline{144}_{H} \times 144_{H}\right)_{45_{1}}=<\Psi_{(-) \mu}^{*}\left|B \Sigma_{\rho \lambda}\right| \Psi_{(+) \mu}><\Psi_{(-) \nu}^{*}\left|B \Sigma_{\rho \lambda}\right| \Psi_{(+) \nu}> \\
\left(\overline{144}_{H} \times 144_{H}\right)_{45_{2}}\left(\overline{144}_{H} \times 144_{H}\right)_{45_{2}}=<\Psi_{(-)[\mu}^{*}|B| \Psi_{(+) \nu]}><\Psi_{(-)[\mu}^{*}|B| \Psi_{(+) \nu]}> \\
\left(\overline{144}_{H} \times 144_{H}\right)_{210}\left(\overline{144}_{H} \times 144_{H}\right)_{210}=<\Psi_{(-) \mu}^{*}\left|B \Gamma_{[\rho} \Gamma_{\sigma} \Gamma_{\lambda} \Gamma_{\xi]}\right| \Psi_{(+) \mu}> \\
\left.\cdot<\Psi_{(-) \nu}^{*} \mid B \Gamma_{[\rho} \Gamma_{\sigma} \Gamma_{\lambda} \Gamma_{\xi]}\right] \Psi_{(+) \nu}>
\end{array}
$$

In the above $\Gamma_{\mu}(\mu=1,2, \ldots, 10)$ are the $S O(10)$ matrices which satisfy the Clifford algebra

$$
\left\{\Gamma_{\mu}, \Gamma_{\nu}\right\}=2 \delta_{\mu \nu}
$$

and $B$ is the $S O(10)$ charge conjugation matrix

$$
B=\prod_{\mu=\text { odd }} \Gamma_{\mu}
$$

The explicit computations are done using the oscillator method[26, 27] and the techniques developed in [24, 28, 29]. These techniques are field theoretic and the $144_{H}\left(\overline{144}_{H}\right)$ plet in this scheme is represented by a constrained vector spinors $\left|\Upsilon_{( \pm) \mu}\right\rangle$ where one imposes the constraint $\Gamma_{\mu} \mid \Upsilon_{( \pm) \mu}>=0$. We carry out an explicit analysis and find

$$
\begin{aligned}
\mathbf{W}_{S B}= & M \mathbf{Q}_{j}^{i} \mathbf{P}_{i}^{j}+\frac{1}{M^{\prime}}\left[-\lambda_{45_{1}}+\frac{1}{6} \lambda_{210}\right] \mathbf{Q}_{j}^{i} \mathbf{P}_{i}^{j} \mathbf{Q}_{l}^{k} \mathbf{P}_{k}^{l} \\
& +\frac{1}{M^{\prime}}\left[-4 \lambda_{45_{1}}-\frac{1}{2} \lambda_{45_{2}}-\lambda_{210}\right] \mathbf{Q}_{k}^{i} \mathbf{P}_{j}^{k} \mathbf{Q}_{l}^{j} \mathbf{P}_{i}^{l},
\end{aligned}
$$


The minimization of $\mathrm{W}_{S B}$ gives

$$
\begin{aligned}
& <\mathbf{Q}_{j}^{i}>=q \operatorname{diag}(2,2,2,-3,-3) \\
& <\mathbf{P}_{j}^{i}>=p \operatorname{diag}(2,2,2,-3,-3),
\end{aligned}
$$

where $q, p$ are constrained by

$$
\frac{M M^{\prime}}{q p}=116 \lambda_{45_{1}}+7 \lambda_{45_{2}}+4 \lambda_{210} .
$$

\section{B. Doublet-triplet splitting}

We discuss now the doublet-triplet splitting. Our general philosophy is that we are working within the context of a landscape scenario where a fine tuning to make the Higgs doublet light is permissible [30, 31]. After spontaneous breaking of the electroweak symmetry discussed in the preceding section, one finds that the part of the superpotential that governs the doublet-triplet splitting is given by

$$
\begin{aligned}
\mathbf{W}_{D T}= & \left\{\frac{4}{5} M+\frac{1}{M^{\prime}}\left(\frac{24}{5} \lambda_{45_{1}}-\frac{4}{15} \lambda_{210}\right)<\mathbf{Q}_{n}^{m}><\mathbf{P}_{m}^{n}>\right\} \mathbf{Q}_{i} \mathbf{P}^{i} \\
& +\left\{\frac{1}{M^{\prime}}\left[-\frac{4}{5} \lambda_{45_{2}}-\frac{32}{15} \lambda_{210}\right]<\mathbf{Q}_{j}^{m}><\mathbf{P}_{m}^{i}>\right\} \mathbf{Q}_{i} \mathbf{P}^{j} \\
& +\left\{M+\frac{1}{M^{\prime}}\left(6 \lambda_{45_{1}}-\frac{1}{3} \lambda_{210}\right)<\mathbf{Q}_{n}^{m}><\mathbf{P}_{m}^{n}>\right\} \mathbf{Q}^{i} \mathbf{P}_{i} \\
& +\left\{\frac{1}{M^{\prime}}\left(\lambda_{45_{2}}\right)<\mathbf{Q}_{i}^{m}><\mathbf{P}_{m}^{j}>\right\} \mathbf{Q}^{i} \mathbf{P}_{j} \\
& +\left\{-\frac{1}{2} M+\frac{1}{M^{\prime}}\left(\lambda_{45_{1}}-\frac{1}{6} \lambda_{210}\right)<\mathbf{Q}_{n}^{m}><\mathbf{P}_{m}^{n}>\right\}\left[\mathbf{Q}_{i j}^{k}+\frac{1}{2 \sqrt{5}}\left(\delta_{i}^{k} \mathbf{Q}_{j}-\delta_{j}^{k} \mathbf{Q}_{i}\right)\right] \\
& \times\left[\mathbf{P}_{k}^{i j}+\frac{1}{2 \sqrt{5}}\left(\delta_{k}^{i} \mathbf{P}^{j}-\delta_{k}^{j} \mathbf{P}^{i}\right)\right] \\
& +\left\{\frac{1}{M^{\prime}}\left(-\frac{1}{2} \lambda_{45_{2}}\right)<\mathbf{Q}_{i}^{m}><\mathbf{P}_{m}^{j}>\right\}\left[\mathbf{Q}_{k l}^{i}+\frac{1}{2 \sqrt{5}}\left(\delta_{k}^{i} \mathbf{Q}_{l}-\delta_{l}^{i} \mathbf{Q}_{k}\right)\right] \\
& \times\left[\mathbf{P}_{j}^{k l}+\frac{1}{2 \sqrt{5}}\left(\delta_{j}^{k} \mathbf{P}^{l}-\delta_{j}^{l} \mathbf{P}^{k}\right)\right] \\
& +\left\{\frac{1}{M^{\prime}}\left[\left(8 \lambda_{45_{1}}-\frac{2}{3} \lambda_{210}\right)<\mathbf{Q}_{m}^{i}><\mathbf{P}_{j}^{m}>\right]\right\}\left[\mathbf{Q}_{i l}^{k}+\frac{1}{2 \sqrt{5}}\left(\delta_{i}^{k} \mathbf{Q}_{l}-\delta_{l}^{k} \mathbf{Q}_{i}\right)\right] \\
& \times\left[\mathbf{P}_{k}^{l j}+\frac{1}{2 \sqrt{5}}\left(\delta_{k}^{l} \mathbf{P}^{j}-\delta_{k}^{j} \mathbf{P}^{l}\right)\right] .
\end{aligned}
$$


In the limit when $\lambda_{45_{1}}=0=\lambda_{45_{2}}, \lambda_{210}=0$, the Higgs doublets and triplets that pair up are the following set

$$
\begin{array}{rrr}
\mathrm{D}_{1}: & \left(\mathbf{Q}^{a}, \mathbf{P}_{a}\right) ; & \mathrm{T}_{1}:\left(\mathbf{Q}^{\alpha}, \mathbf{P}_{\alpha}\right) \\
\mathrm{D}_{2}: & \left(\mathbf{Q}_{a}, \mathbf{P}^{a}\right) ; & \mathrm{T}_{2}:\left(\mathbf{Q}_{\alpha}, \mathbf{P}^{\alpha}\right) \\
\mathrm{D}_{3}: & \left(\widetilde{\mathbf{Q}}_{a}, \widetilde{\mathbf{P}}^{a}\right) ; & \mathrm{T}_{3}:\left(\widetilde{\mathbf{Q}}_{\alpha}, \widetilde{\mathbf{P}}^{\alpha}\right) \\
\mathrm{T}_{4}:\left(\widetilde{\mathbf{Q}}^{\alpha}, \widetilde{\mathbf{P}}_{\alpha}\right) . &
\end{array}
$$

Here $\mathrm{T}_{1}, \mathrm{~T}_{2}, \mathrm{~T}_{3}$ are color triplet (anti-triplet) pairs which have charges $Q=-1 / 3(1 / 3)$, while $\mathrm{T}_{4}$ have charges $Q=-4 / 3(4 / 3)$. Thus the pattern of color Higgs multiplets discussed in Sec. III is reproduced here.

Mixings among the mutiplets occur when the couplings $\lambda_{45_{1}}, \lambda_{45_{2}}, \lambda_{210}$ are non-zero. For the Higgs doublet fields the doublet $D_{1}$ is decoupled while the mixings that lead to the doublets $D_{2}$ and $D_{3}$ are generated by the mass matrix

$$
\widetilde{\mathbf{P}}^{\mathbf{a}}\left[\begin{array}{cc}
\mathbf{Q}_{a} & \widetilde{\mathbf{Q}}_{a} \\
\sqrt{\frac{3}{5} \frac{q p}{M^{\prime}}\left(10 \lambda_{45_{1}}+\frac{5}{4} \lambda_{45_{2}}-\frac{5}{6} \lambda_{210}\right)} & -\frac{1}{2} M+\frac{q p}{M^{\prime}}\left(-74 \lambda_{45_{1}}-\frac{31}{4} \lambda_{45_{2}}+\frac{7}{6} \lambda_{210}\right)
\end{array}\right]
$$

For the triplets, $T_{1}$ and $T_{4}$ are decoupled while $T_{2}$ and $T_{3}$ mix. The mixings that lead to the Higgs triplets $T_{2}$ and $T_{3}$ are given by the mass matrix

$$
\begin{aligned}
& \begin{array}{ll}
\mathbf{Q}_{\alpha} & \widetilde{\mathbf{Q}}_{\alpha}
\end{array} \\
& \widetilde{\mathbf{P}}^{\alpha}\left[\begin{array}{cc}
\frac{3}{5} M+\frac{q p}{M^{\prime}}\left(\frac{696}{5} \lambda_{45_{1}}-\frac{9}{2} \lambda_{45_{2}}-\frac{257}{15} \lambda_{210}\right) & \frac{\sqrt{5}}{2} \frac{q p}{M^{\prime}}\left(8 \lambda_{45_{1}}+\lambda_{45_{2}}-\frac{2}{3} \lambda_{210}\right) \\
\frac{\sqrt{5}}{2} \frac{q p}{M^{\prime}}\left(8 \lambda_{45_{1}}+\lambda_{45_{2}}-\frac{2}{3} \lambda_{210}\right) & -M+\frac{q p}{M^{\prime}}\left(-24 \lambda_{45_{1}}-\frac{13}{2} \lambda_{45_{2}}-3 \lambda_{210}\right)
\end{array}\right] .
\end{aligned}
$$


We represent the mass eigenstates by primed fields, and the primed fields may be expressed in terms of the unprimed ones through the following transformation matrices

$$
\begin{gathered}
{\left[\begin{array}{c}
\left(\mathbf{Q}_{a}^{\prime}, \mathbf{P}^{\prime a}\right) \\
\left(\widetilde{\mathbf{Q}}_{a}^{\prime}, \widetilde{\mathbf{P}}^{\prime a}\right)
\end{array}\right]=\left[\begin{array}{cc}
\cos \vartheta_{\mathrm{D}} & \sin \vartheta_{\mathrm{D}} \\
-\sin \vartheta_{\mathrm{D}} & \cos \vartheta_{\mathrm{D}}
\end{array}\right]\left[\begin{array}{c}
\left(\mathbf{Q}_{a}, \mathbf{P}^{a}\right) \\
\left(\widetilde{\mathbf{Q}}_{a}, \widetilde{\mathbf{P}}^{a}\right)
\end{array}\right]} \\
{\left[\begin{array}{c}
\left(\mathbf{Q}_{\alpha}^{\prime}, \mathbf{P}^{\prime \alpha}\right) \\
\left(\widetilde{\mathbf{Q}}_{\alpha}^{\prime}, \widetilde{\mathbf{P}}^{\prime \alpha}\right)
\end{array}\right]=\left[\begin{array}{cc}
\cos \vartheta_{\mathrm{T}} & \sin \vartheta_{\mathrm{T}} \\
-\sin \vartheta_{\mathrm{T}} & \cos \vartheta_{\mathrm{T}}
\end{array}\right]\left[\begin{array}{c}
\left(\mathbf{Q}_{\alpha}, \mathbf{P}^{\alpha}\right) \\
\left(\widetilde{\mathbf{Q}}_{\alpha}, \widetilde{\mathbf{P}}^{\alpha}\right)
\end{array}\right],}
\end{gathered}
$$

where

$$
\begin{aligned}
& \tan \vartheta_{\mathrm{D}}=\frac{1}{\mathrm{~d}_{3}}\left(\mathrm{~d}_{2}+\sqrt{\mathrm{d}_{2}^{2}+\mathrm{d}_{3}^{2}}\right) \\
& \tan \vartheta_{\mathrm{T}}=\frac{1}{\mathrm{t}_{3}}\left(\mathrm{t}_{2}+\sqrt{\mathrm{t}_{2}^{2}+\mathrm{t}_{3}^{2}}\right),
\end{aligned}
$$

and where

$$
\begin{aligned}
\mathrm{d}_{1} & =-\frac{2}{5} M+\frac{q p}{M^{\prime}}\left(\frac{296}{5} \lambda_{45_{1}}-16 \lambda_{45_{2}}-\frac{392}{15} \lambda_{210}\right) \\
\mathrm{d}_{2} & =-\frac{8}{5} M+\frac{q p}{M^{\prime}}\left(-\frac{1036}{5} \lambda_{45_{1}}+\frac{1}{2} \lambda_{45_{2}}+\frac{427}{15} \lambda_{210}\right) \\
\mathrm{d}_{3} & =2 \sqrt{\frac{3}{5}} \frac{q p}{M^{\prime}}\left(10 \lambda_{45_{1}}+\frac{5}{4} \lambda_{45_{2}}-\frac{5}{6} \lambda_{210}\right) \\
\mathrm{t}_{1} & =-\frac{2}{5} M+\frac{q p}{M^{\prime}}\left(\frac{576}{5} \lambda_{45_{1}}-11 \lambda_{45_{2}}-\frac{302}{15} \lambda_{210}\right) \\
\mathrm{t}_{2} & =-\frac{8}{5} M+\frac{q p}{M^{\prime}}\left(-\frac{816}{5} \lambda_{45_{1}}-2 \lambda_{45_{2}}+\frac{212}{15} \lambda_{210}\right) \\
\mathrm{t}_{3} & =\sqrt{5} \frac{q p}{M^{\prime}}\left(8 \lambda_{45_{1}}+\lambda_{45_{2}}-\frac{2}{3} \lambda_{210}\right) .
\end{aligned}
$$

The mass eigenvalues are found to be

$$
\begin{aligned}
M_{\mathrm{D}_{1}} & =M+\frac{q p}{M^{\prime}}\left(180 \lambda_{45_{1}}+9 \lambda_{45_{2}}-10 \lambda_{210}\right) \\
M_{\mathrm{D}_{2}, \mathrm{D}_{3}} & =\frac{1}{2}\left(\mathrm{~d}_{1} \pm \sqrt{\mathrm{d}_{2}^{2}+\mathrm{d}_{3}^{2}}\right)
\end{aligned}
$$

and

$$
\begin{aligned}
M_{\mathrm{T}_{1}} & =M+\frac{q p}{M^{\prime}}\left(180 \lambda_{45_{1}}+4 \lambda_{45_{2}}-10 \lambda_{210}\right) \\
M_{\mathrm{T}_{4}} & =-M+\frac{q p}{M^{\prime}}\left(-84 \lambda_{45_{1}}-4 \lambda_{45_{2}}+2 \lambda_{210}\right) \\
M_{\mathrm{T}_{2}, \mathrm{~T}_{3}} & =\frac{1}{2}\left(\mathrm{t}_{1} \pm \sqrt{\mathrm{t}_{2}^{2}+\mathrm{t}_{3}^{2}}\right) .
\end{aligned}
$$

The above allow for making one pair of Higgs doublets light by a constraint while all the Higgs triplets remain heavy. 


\section{Matter-Higgs interactions}

The 16-plet of matter can interact with 144-plet of Higgs only via quartic couplings. Here we consider the following interactions

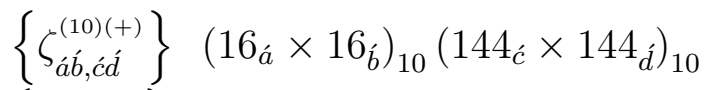

$$
\begin{aligned}
& \left\{\xi_{a b \dot{a}, \dot{c} \dot{d}}^{(10)+(+)}\right\} \quad\left(16_{\dot{a}} \times 16_{\dot{b}}\right)_{10}\left(\overline{144}_{\dot{c}} \times \overline{144}_{\dot{d}}\right)_{10} \\
& \left\{\varrho_{a b b, \dot{c} d}^{(126, \overline{126})(+)}\right\}\left(16_{\dot{a}} \times 16_{\dot{b}}\right)_{\overline{126}}\left(144_{\dot{c}} \times 144_{\dot{d}}\right)_{126} \\
& \left\{\lambda_{a \dot{a} \dot{c} \dot{c} \dot{d}}^{(45)}\right\}\left(16_{\dot{a}} \times \overline{144}_{\dot{b}}\right)_{45}\left(16_{\dot{c}} \times \overline{144}_{\dot{d}}\right)_{45}, \\
& \left\{\zeta_{a ́ b, c ́ d}^{(120)(-)}\right\}\left(16_{a} \times 16_{\dot{b}}\right)_{120}\left(144_{\dot{c}} \times 144_{\dot{d}}\right)_{120} \\
& \left\{\xi_{a \dot{a} b, \dot{c} d}^{(12)(-)}\right\} \quad\left(16_{\dot{a}} \times 16_{\dot{b}}\right)_{120}\left(\overline{144}_{\dot{c}} \times \overline{144}_{\dot{d}}\right)_{120} \\
& \left\{\lambda_{a \dot{a}, \dot{c} \dot{d}}^{(54)}\right\}\left(16_{\dot{a}} \times \overline{144}_{\dot{b}}\right)_{54}\left(16_{\dot{c}} \times \overline{144}_{\dot{d}}\right)_{54} \\
& \left\{\lambda_{\dot{a} b, \dot{c} d}^{(10)}\right\}\left(16_{\dot{a}} \times 144_{\dot{b}}\right)_{10}\left(16_{\dot{c}} \times 144_{\dot{d}}\right)_{10},
\end{aligned}
$$

which contribute to the masses of quarks and leptons. Since these couplings are quartic they are Planck scale suppressed. We assume that the first two generation masses arise from such couplings while the third generation masses arise from cubic interactions. The quantities within \{\} are parameters associated with the particular quartic couplings with which they appear. The matter-Higgs quartic couplings can be decomposed in $S U(5)$ representations as follows

$$
W_{4}=\sum_{i=1}^{5} W_{4}^{(i)} .
$$

The explicit analysis of the couplings in its $S U(5) \times U(1)$ decomposed form is carrried out using oscillator method [26, 27] and the techniques developed in [24, 28, 29]. The result of the analysis is recorded in Appendix A. It was noted in [25] that much larger masses for the third generation can be obtained if one allows for the mixings of the 16 plets of matter in the third generation with 10 and 45 plets of matter. Thus one may have cubic couplings of the type

$$
\left(16.10 .144_{H}\right), \quad\left(16.45 \cdot \overline{144}_{H}\right) .
$$

We note in passing that the particle content of 10 and 45 of matter in its $S U(2) \times$ $S U(3)_{C} \times U(1)$ decomposition is as follows: for the 10 plet of matter we have $10=$ $(1,1)(6)+(1, \overline{3})(-4)+(2,3)(1)$ while the 45 -plet has the decomposition $45=(2,1)(3)+$ 
$(1,3)(-2)+(3,3)(-2)+(1, \overline{3})(8)+(2, \overline{3})(-7)+(1, \overline{6})(-2)+(2,8)(3)$. An explicit compu-

tation of the couplings in $S U(5) \times U(1)$ decomposition using the techniques of [24, 28, 29] gives

$\begin{aligned} \mathbf{W}^{16 \times \overline{144} \times 45} & =f_{a ́ b}^{(45)}\left[\frac{1}{\sqrt{10}} \epsilon_{i j k l m} \mathbf{M}_{a}^{i j} \mathbf{P}^{k} \mathbf{F}_{\hat{b}}^{(45) l m}+\frac{1}{\sqrt{2}} \epsilon_{i j k l m} \mathbf{M}_{a}^{i j} \mathbf{P}_{n}^{k l} \mathbf{F}_{\hat{b}}^{(45) m n}-2 \sqrt{2} \mathbf{M}_{a i} \mathbf{P}_{j} \mathbf{F}_{b}^{(45) i j}+\ldots\right] \\ \mathbf{W}^{16 \times 144 \times 10} & =f_{a ́ b}^{(10)}\left[-\frac{1}{2 \sqrt{10}} \mathbf{M}_{a}^{i j} \mathbf{Q}_{j} \mathbf{F}_{b i}^{(10)}+\frac{1}{2 \sqrt{2}} \mathbf{M}_{a}^{i j} \mathbf{Q}_{i j}^{k} \mathbf{F}_{b k}^{(10)}+\ldots\right] .\end{aligned}$

Using the above interactions, one can generate a realistic model of quark-lepton-neutrino textures. However, a detailed analysis of the textures generated by the interactions above and fits to the experimental data is outside the scope of this work. Here we focus on the baryon and lepton number violating dimension five operators generated by the interactions above and how they can be suppressed consistent with the current data.

\section{Baryon and lepton number violating dimension-5 operators}

Using Eqs.(53), (54), and (43) and inserting mass terms for triplets responsible for proton decay, we find

$$
\begin{aligned}
\mathbf{W}_{B \& L}= & J_{1}^{\alpha} \mathbf{P}_{\alpha}+K_{1 \alpha} \mathbf{Q}^{\alpha}+M_{\mathrm{T}_{1}} \mathbf{Q}^{\alpha} \mathbf{P}_{\alpha} \\
& +\left[J_{2 \alpha} \cos \vartheta_{\mathbf{T}}+J_{3 \alpha} \sin \vartheta_{\mathrm{T}}\right] \mathbf{P}^{\prime \alpha}+\left[K_{2}^{\alpha} \cos \vartheta_{\mathrm{T}}+K_{3}^{\alpha} \sin \vartheta_{\mathrm{T}}\right] \mathbf{Q}_{\alpha}^{\prime}+M_{\mathrm{T}_{2}} \mathbf{Q}_{\alpha}^{\prime} \mathbf{P}^{\prime \alpha} \\
& +\left[-J_{2 \alpha} \sin \vartheta_{\mathrm{T}}+J_{3 \alpha} \cos \vartheta_{\mathrm{T}}\right] \widetilde{\mathbf{P}}^{\prime \alpha}+\left[-K_{2}^{\alpha} \sin \vartheta_{\mathrm{T}}+K_{3}^{\alpha} \cos \vartheta_{\mathrm{T}}\right] \widetilde{\mathbf{Q}}_{\alpha}^{\prime}+M_{\mathrm{T}_{3}} \widetilde{\mathbf{Q}}_{\alpha}^{\prime} \widetilde{\mathbf{P}}^{\prime \alpha} \\
& +J_{4}^{\alpha} \widetilde{\mathbf{P}}_{\alpha}^{\prime}+K_{4 \alpha} \widetilde{\mathbf{Q}}^{\prime \alpha}+M_{\mathrm{T}_{4}} \widetilde{\mathbf{Q}}^{\prime \alpha} \widetilde{\mathbf{P}}_{\alpha}^{\prime} .
\end{aligned}
$$


Here we have defined

$$
\begin{aligned}
& J_{1}^{\alpha}=2 p\left[4\left(4 \xi_{\dot{a} \dot{b}}^{(10)(+)}-\lambda_{a, b}^{(45)}\right)\left(\epsilon^{\alpha \beta \gamma} \mathbf{D}_{L \dot{a} \beta}^{c} \mathbf{U}_{L \dot{b} \gamma}^{c}\right)+\left(-16 \xi_{\dot{a} \dot{b}}^{(10)(+)}-\lambda_{a, b}^{(45)}+5 \lambda_{a, b}^{(54)}\right)\left(\mathbf{E}_{L \dot{a}} \mathbf{U}_{L \dot{b}}^{\alpha}+\nu_{L \dot{a}} \mathbf{D}_{L \dot{b}}^{\alpha}\right)\right] \\
& -2 \sqrt{2} f_{33}^{(45)}\left(-\epsilon^{\alpha \beta \gamma\left(\overline{5}_{16}\right)} \mathbf{b}_{L \beta}^{\mathrm{c}}{ }^{\left(10_{45}\right)} \mathbf{t}_{L \gamma}^{\mathrm{c}}+{ }^{\left(\overline{5}_{16}\right)} \boldsymbol{\tau}_{L}{ }^{\left(10_{45}\right)} \mathbf{t}_{L}^{\alpha}+{ }^{\left(\overline{5}_{16}\right)} \boldsymbol{\nu}_{L \tau}{ }^{\left(10_{45}\right)} \mathbf{b}_{L}^{\alpha}\right), \\
& K_{1 \alpha}=32 q\left(\zeta_{\dot{a} \dot{b}}^{(10)(+)}+\frac{2}{15} \varrho_{\dot{a} \dot{b}}^{(126, \overline{126})(+)}\right)\left(\mathbf{U}_{L a \dot{\alpha}}^{c} \mathbf{E}_{L \dot{b}}^{c}-\epsilon_{\alpha \beta \gamma} \mathbf{U}_{L \dot{a}}^{\beta} \mathbf{D}_{L \dot{b}}^{\gamma}\right)
\end{aligned}
$$

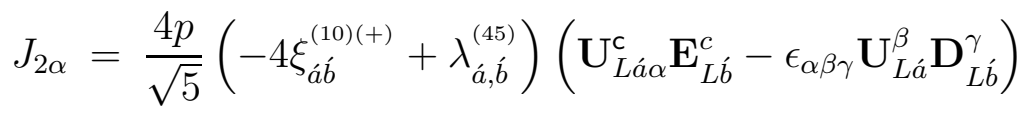

$$
\begin{aligned}
& +2 \sqrt{\frac{2}{5}} f_{33}^{(45)}\left({ }^{\left(10_{16}\right)} \mathbf{t}_{L \alpha}^{\mathrm{c}}{ }^{\left(10_{45}\right)} \boldsymbol{\tau}_{L}^{c}+{ }^{\left({ }^{(10}{ }_{16}\right)} \boldsymbol{\tau}_{L}^{c}{ }^{\left(10_{45}\right)} \mathbf{t}_{L \alpha}^{\mathrm{c}}+\epsilon_{\alpha \beta \gamma}{ }^{\left(10_{16}\right)} \mathbf{t}_{L}^{\beta}{ }^{\left(10_{45}\right)} \mathbf{b}_{L}^{\gamma}-\epsilon_{\alpha \beta \gamma}{ }^{\left(10_{16}\right)} \mathbf{b}_{L}^{\beta}{ }^{\left(10_{45}\right)} \mathbf{t}_{L}^{\gamma}\right),
\end{aligned}
$$

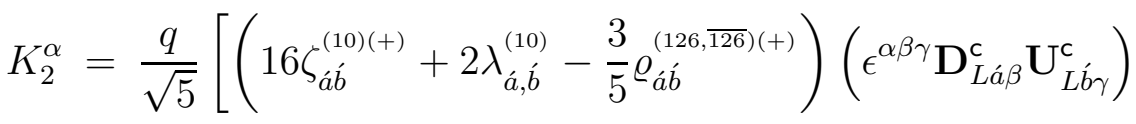

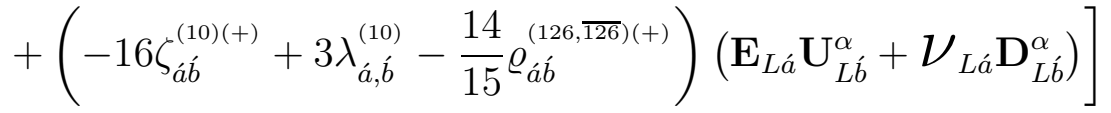

$$
\begin{aligned}
& -\frac{1}{2 \sqrt{10}} f_{33}^{(10)}\left(\epsilon^{\alpha \beta \gamma\left({ }^{(1016}\right)} \mathbf{t}_{L \beta}^{\mathrm{c}}{ }^{\left(\overline{5}_{10}\right)} \mathbf{b}_{L \gamma}^{\mathrm{c}}+{ }^{\left(10_{16}\right)} \mathbf{t}_{L}^{\alpha}{ }^{\left(\overline{5}_{10}\right)} \boldsymbol{\tau}_{L}+{ }^{\left(10_{16}\right)} \mathbf{b}_{L}^{\alpha}{ }^{\left(\overline{5}_{10}\right)} \boldsymbol{\nu}_{L \tau}\right),
\end{aligned}
$$

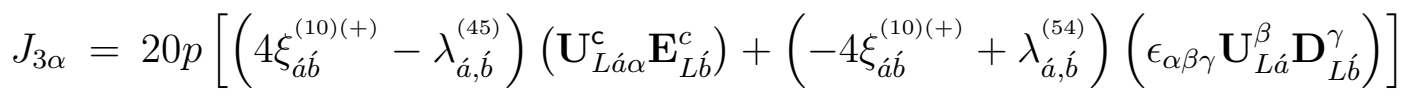

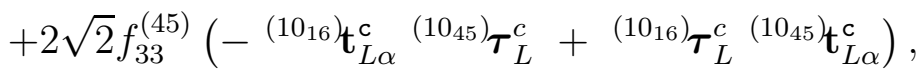

$$
\begin{aligned}
& K_{3}^{\alpha}=q\left[\left(-80 \zeta_{a ́ b}^{(10)(+)}+\frac{3}{5} \varrho_{a}^{(126, \overline{126})(+)}\right)\left(\epsilon^{\alpha \beta \gamma} \mathbf{D}_{L \dot{a} \beta}^{c} \mathbf{U}_{L \dot{b} \gamma}^{\mathrm{c}}\right)\right. \\
& \left.+2\left(40 \zeta_{a ́ b}^{(10)(+)}-\frac{1}{15} \varrho_{a ́ b}^{(126, \overline{126)(+)}}\right)\left(\mathbf{E}_{L \dot{a}} \mathbf{U}_{L \dot{b}}^{\alpha}+\nu_{L \dot{a}} \mathbf{D}_{L \dot{b}}^{\alpha}\right)\right] \\
& +\frac{1}{2 \sqrt{2}} f_{33}^{(10)}\left(\epsilon^{\alpha \beta \gamma\left(10_{16}\right)} \mathbf{t}_{L \beta}^{\mathrm{c}}{ }^{\left(\overline{5}_{10}\right)} \mathbf{b}_{L \gamma}^{\mathrm{c}}-{ }^{\left(10_{16}\right)} \mathbf{t}_{L}^{\alpha}{ }^{\left(\overline{5}_{10}\right)} \boldsymbol{\tau}_{L}-{ }^{\left(10_{16}\right)} \mathbf{b}_{L}^{\alpha\left(\overline{5}_{10}\right)} \boldsymbol{\nu}_{L \tau}\right),
\end{aligned}
$$

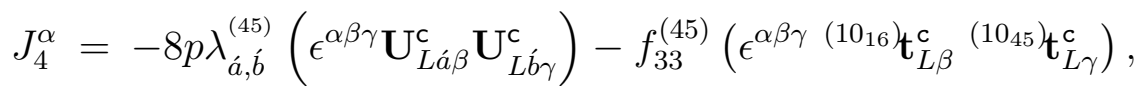

$$
\begin{aligned}
& K_{4 \alpha}=-\frac{16 q}{15} \varrho_{a}^{(126, \overline{126})(+)}\left(\mathbf{D}_{L a \alpha}^{c} \mathbf{E}_{L \dot{b}}^{c}\right)+\frac{1}{\sqrt{2}} f_{33}^{(10)}\left({ }^{\left(10_{16}\right)} \boldsymbol{\tau}_{L}^{c}\left(\overline{5}_{10} \mathbf{b}_{L \alpha}^{c}\right)\right.
\end{aligned}
$$

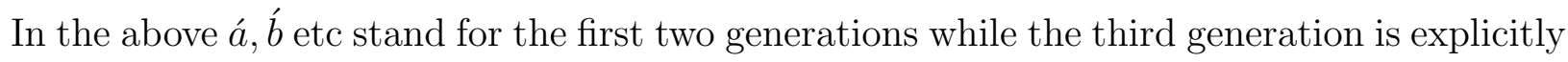
factored out and the fields denoted by their familiar symbols $\mathbf{b}, \mathbf{t}, \tau$ for the bottom quark, top quark and for the $\tau$ lepton. We note that the currents $J_{4}$ and $K_{4}$ are similar to the tilde 
currents $\tilde{J}$ and $\tilde{K}$ discussed in Sec. III. Finally, integrating out the Higgs triplet fields in Eq.(44), we obtain the usual $R R R R$ and $L L L L$ operators. These are exhibited in Appendix B.

We now explore the conditions under which proton decay is suppressed. First all the terms which contain tau do not contribute since proton cannot decay into a final state with a tau. It is now easily checked that all the remaining $L L L L$ and $R R R R$ terms do not contribute or cancel under the constraints

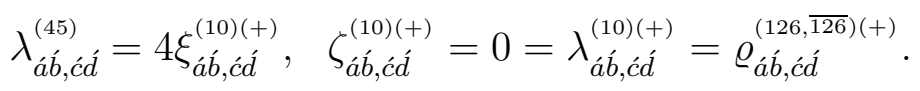

The cancellation condition of Eq.(46) involves the quartic couplings and thus the Planck scale effects. A cancellation to reduce the proton decay amplitude by a factor of ten will lead to extending the proton life time by a factor $10^{2}$ and may be sufficient to suppress proton decay to the current level of experiment for most models making them phenomenologically viable. At the same time it leaves open the possibility that proton decay may be observed in the next round of experiment[32]. For the case when the $Q=-1 / 3(1 / 3)$ Higgsino exchange is much more suppressed than the $Q=-4 / 3(4 / 3)$ Higgsino exchange, there will be only $R R R R$ type operators and the dominant mode would be $\bar{\nu}_{\tau} \bar{K}^{+}$.

\section{E. quark-lepton textures}

The analysis presented above produces the quark lepton textures with the correct sizes. The first two generation of masses arise in the above model from quartic couplings of matter fields with the 144 and $\overline{144}$ Higgs fields[24], while the third generation masses arise from cubic couplings involving 144 and $\overline{144}$ of Higgs and additional 10 and 45 plets of $S O(10)$ matter fields [25]. Below we show how the quartic couplings can generate the desired sizes for the up quark, down quark, lepton and neutirno masses for the first two generations. Using the decomposition of $S O(10)$ couplings in terms of $S U(5)$ couplings the up-quark masses arise from the interactions

$$
10_{M} 10_{M} \frac{24_{H}}{M} 5_{H}
$$

where the fields above are all in $S U(5)$ representations. Similarly the down quark and lepton masses arise from the interaction

$$
10_{M} \overline{5}_{M} \frac{24_{H}}{M} \overline{5}_{H}
$$


The $R R, L R$ and $L L$ neutrino masses arise from the following terms

$$
\begin{array}{r}
\mathrm{RR}-\nu \text { mass : } 1_{M} 1_{M} \frac{24_{H}}{M} 24_{H} \\
\mathrm{LR}-\nu \text { mass }: \quad \overline{5}_{M} 1_{M} \frac{24_{H}}{M}\left(5_{H}, 45_{H}\right) \\
\text { LL }-\nu \text { mass }: \quad \overline{5}_{M} \overline{5}_{M} \frac{5_{H}}{M} 5_{H}
\end{array}
$$

It is now easily seen that the right sizes for the quark-lepton masses for the first two generations can appear after $24_{H}, 5_{H}+\overline{5}_{H}, 45_{H}+\overline{45}_{H}$ develop vacuum expectation values. Additionally for the third generation one has cubic couplings which can generate relatively large masses typical of third generation. The full analysis is rather involved and is outside the scope of this paper.

\section{CONCLUSION}

In this paper we have discussed the mechanism where contributions from different operators that contribute to the baryon and lepton number violating dimension 5 operators tend to cancel producing an enhancement for the proton decay lifetime in supersymmetric unified theories. The cancellation mechanism works when there are more than one pair of Higgs triplets generating baryon and lepton number violating interactions, with their Yukawa coupling having similar generational symmetry. We have discussed in this paper three specific examples, two for the $S U(5)$ case and the other for the $S O(10)$ case. For the $S U(5)$ case we first considered a model with a Higgs sector consisting of $5_{H}+\overline{5}_{H}, 24_{H}$, and $45_{H}+\overline{45}_{H}$ plets of Higgs. Here the $24_{H}$ plet breaks the GUT symmetry down to $S U(3)_{C} \times S U(2)_{L} \times U(1)_{Y}$, and the Higgs doublets from the $5_{H}+\overline{5}_{H}$ enter in the electroweak symmetry breaking, while the Higgs triplet fields from $5_{H}+\overline{5}_{H}$ and from $45_{H}+\overline{45}_{H}$ generate baryon and lepton number violating interactions. It is then shown that the baryon and lepton number violating contributions arising from the exchange of Higgs triplets from the $45_{H}+\overline{45}_{H}$ can cancel the baryon and lepton number contributions arising from the Higgs triplet exchange from the $5_{H}+\overline{5}_{H}$ when the generational dependence of the Yukawa couplings of the $5_{H}+\overline{5}_{H}$ and of $45_{H}+\overline{45}_{H}$ are

similar. Next we considered an $S U(5)$ example with only $5_{H}+\overline{5}_{H}$ and $24_{H}$ of Higgs but including Planck scale contributions. Here it is seen that one can produce the appropriate 
quark-lepton textures and a complete suppression of B\&L violating dimension five operators.

For the $S O(10)$ case, we consider a recently proposed model where a one step breaking of $S O(10)$ to $S U(3)_{C} \times U(1)_{e m}$ can occur with a $144_{H}+\overline{144}_{H}$ of Higgs. Here the decomposition of $144_{H}+\overline{144}_{H}$ contains automatically $45_{H}+\overline{45}_{H}$ of Higgs in addition to $5_{H}+\overline{5}_{H}$ of Higgs. The interactions of $144_{H}+\overline{144}_{H}$ with matter are at least quartic, but normal Yukawa type coupling arise after spontaneous breaking when $144_{H}+\overline{144}_{H}$ develop VEVs. In addition large 3rd generation masses can arise with cubic interactions when $10+45$ of matter is included. The analysis including all these interactions was carried out and baryon and lepton number violating dimension 5 operators were computed. It is then found that simple constraints suppress all LLLL and RRRR baryon and lepton number violating dimension five operators. While we have illustrated the mechanism for three models, it is likely applicable to a larger class in which the baryon and lepton number violating operators arise from more than one source. The cancellation mechanism can allow for a complete or partial suppression of proton decay, allowing for suppression consistent with the current experimental limits while allowing for the possibility that proton decay may become visible in the next round of nucleon stability experiments[32]. Finally, we have not addressed in this work issues related to mass spectra for the heavy fields, gauge coupling unification, and a detailed numerical fit to quark-lepton-neutrino mass textures. A detailed analysis of these topics is outside the scope of this paper. These topics are worthy of further investigations.

Finally we note that recently there has been much further work on the interface of GUTs and strings (see, e.g., Ref.[33] and the references therein) which make progress towards the generation of realistic particle physics models. While some models are free of B\&L violating dimension five operators, others are not[33], and the cancellation mechanism may play a role in making such models viable. More specifically, a class of string models which would otherwise be eliminated by the experimental constraint on B\&L violating dimension five operators could become phenomenology admissible using the cancellation mechanism proposed here. Further, as noted in Sec.(II) the quantum gravity corrections which were introduced in Eqs.(4) and (5) could also be utilized for the suppression of dimension five proton decay. However, a detailed analysis of this phenomenon is outside the scope of the this paper. 


\section{Acknowledgments}

Correspondence with K.S. Babu and Ilia Gogoladze in early stage of the work is acknowledged. This work is supported in part by NSF grant PHY-0456568.

\section{APPENDICES}

\section{A. Matter -Higgs quartic couplings}

In this appendix we give further details of the matter-higgs quartic couplings discussed in Sec.(VC). Below we exhibit the results for $W_{4}^{(i)}(\mathrm{i}=1-5)$ that appear in Sec.(VC). Our analysis gives

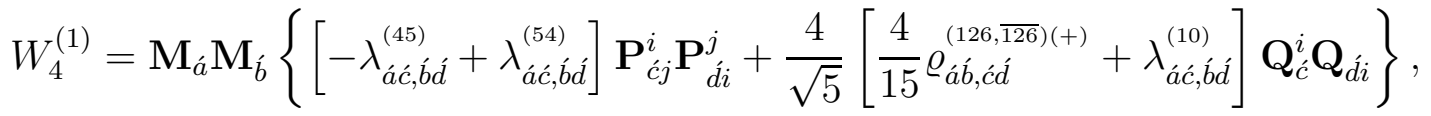

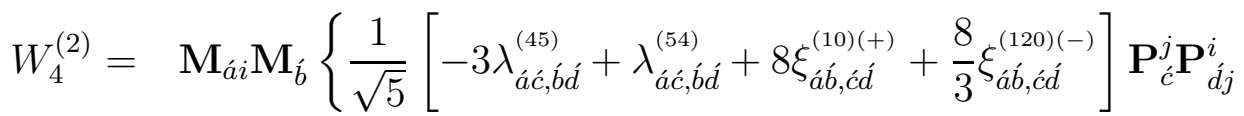

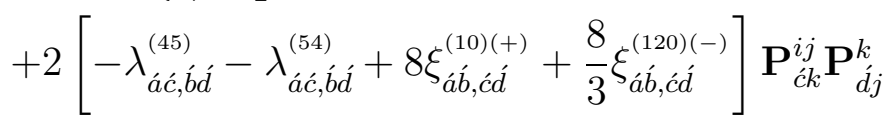

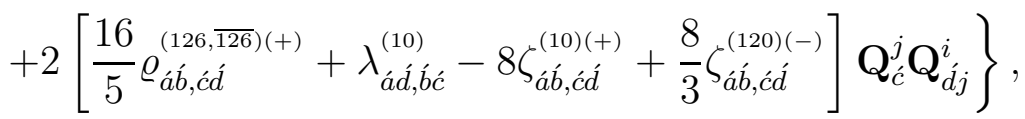

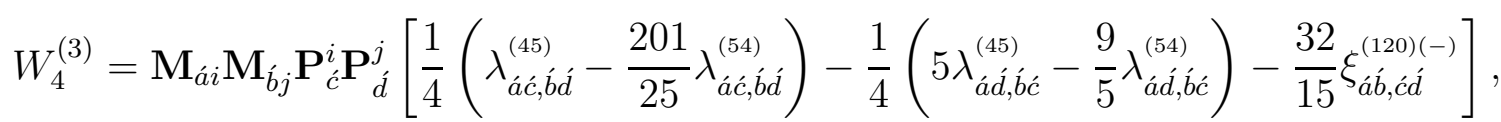

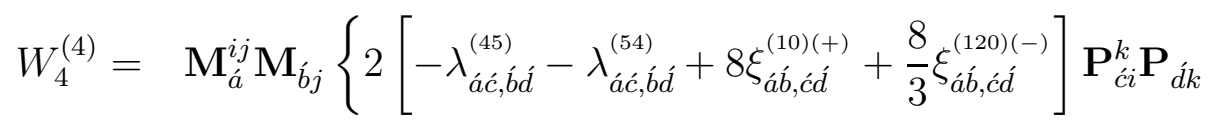

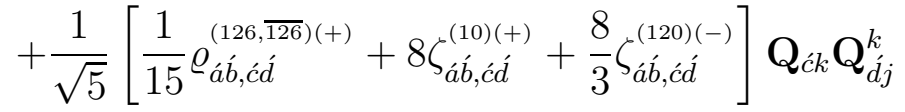

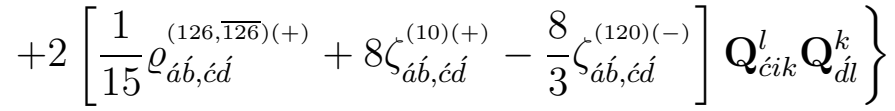

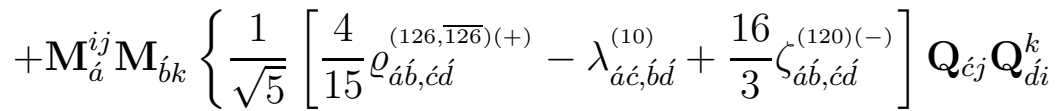

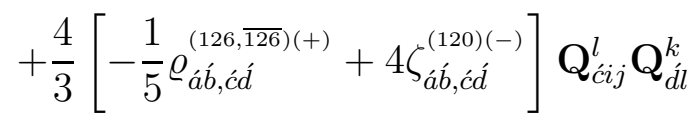

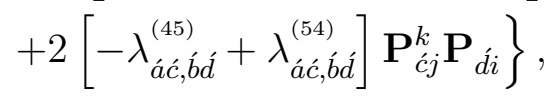




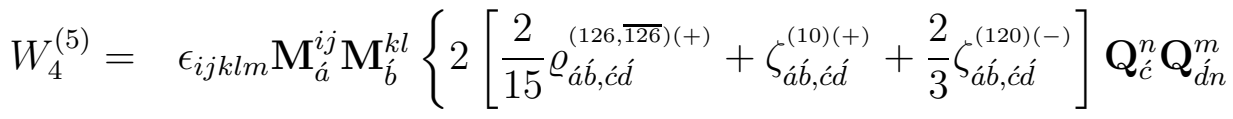

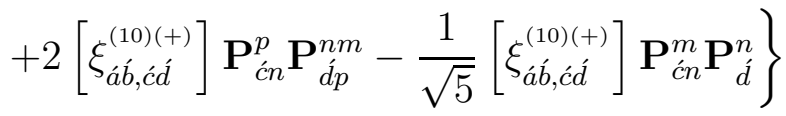

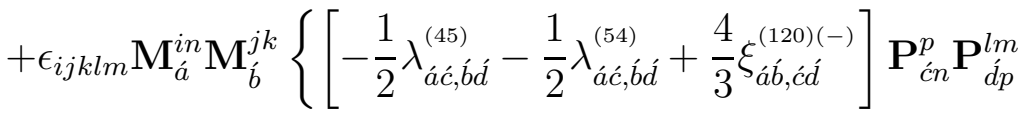

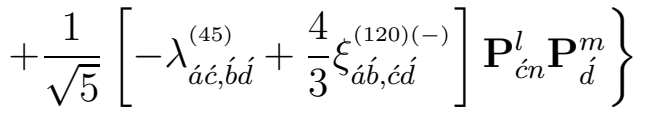

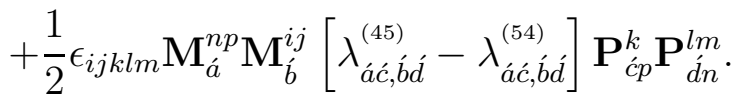

\section{B. Analysis of LLLL and RRRR dimension five operators}

Below we exhibit the result of the analysis of LLL and RRRR dimension five operators. These dimension five operators are gotten by integration over all the Higgs triplet fields.

$$
\mathrm{W}_{B \& L}^{d i m-5}=\sum_{g=I}^{I I I}\left(\mathrm{~W}_{\mathrm{R}}^{(g)}+\mathrm{W}_{\mathrm{L}}^{(g)}\right)
$$


The index $g$ above denotes whether the particular operator connects one $(I)$, two $(I I)$ or three $(I I I)$ generations of fermions. The operators in Eq.(55) are defined through

$$
\begin{aligned}
& \mathrm{W}_{\mathrm{R}}^{(I)}=\mathrm{R}^{(I)} \quad \epsilon^{\alpha \beta \gamma\left(\overline{5}_{16}\right)} \mathbf{b}_{L \alpha}^{\mathrm{c}}{ }^{\left(10_{16}\right)} \mathbf{t}_{L \beta}^{\mathrm{c}}{ }^{\left(10_{16}\right)} \mathbf{t}_{L \gamma}^{\mathrm{c}}{ }^{\left(10_{16}\right)} \boldsymbol{\tau}_{L}^{c},
\end{aligned}
$$

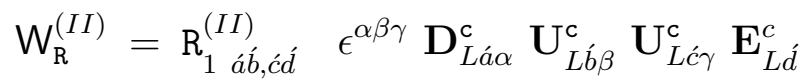

$$
\begin{aligned}
& +\mathrm{R}_{2}^{(I I) \dot{a} b \dot{c} \dot{c} \dot{d}} \quad \epsilon^{\alpha \beta \gamma} \mathbf{D}_{L \dot{\alpha} \alpha}^{c} \mathbf{E}_{L \dot{b}}^{c} \mathbf{U}_{L \dot{c} \beta}^{c} \mathbf{U}_{L \dot{d} \gamma}^{c}, \\
& \mathrm{~W}_{\mathrm{R}}^{(I I I)}=\mathrm{R}_{1 \dot{a} \dot{b}}^{(I I I)} \quad \epsilon^{\alpha \beta \gamma}{ }^{\left(5_{16}\right)} \mathbf{b}_{L \alpha}^{c}{ }^{\left(10_{16}\right)} \mathbf{t}_{L \beta}^{c} \mathbf{U}_{L \dot{a} \gamma}^{c} \mathbf{E}_{L \dot{b}}^{c}
\end{aligned}
$$

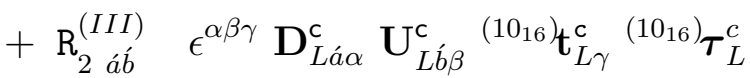

$$
\begin{aligned}
& +\mathrm{R}_{3 \dot{a} \dot{b}}^{(I I I)} \epsilon^{\alpha \beta \gamma}{ }^{\left(5_{16}\right)} \mathbf{b}_{L \alpha}^{c}{ }^{\left(10_{16}\right)} \boldsymbol{\tau}_{L}^{c} \mathbf{U}_{L \dot{a} \beta}^{c} \mathbf{U}_{L \dot{b} \gamma}^{c} \\
& +\mathrm{R}_{4 \dot{a} \dot{b}}^{(I I I)} \quad \epsilon^{\alpha \beta \gamma} \mathbf{D}_{L a ́ \alpha}^{c} \mathbf{E}_{L \dot{b}}^{c}{ }^{\left(10_{16}\right)} \mathbf{t}_{L \beta}^{c}{ }^{\left(10_{16}\right)} \mathbf{t}_{L \gamma}^{c},
\end{aligned}
$$

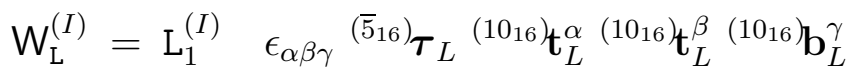

$$
\begin{aligned}
& +\mathrm{L}_{2}^{(I)} \epsilon_{\alpha \beta \gamma}{ }^{\left(5_{16}\right)} \boldsymbol{\nu}_{L \tau}{ }^{\left(10_{16}\right)} \mathbf{b}_{L}^{\alpha}{ }^{\left(10_{16}\right)} \mathbf{t}_{L}^{\beta}{ }^{\left(10_{16}\right)} \mathbf{b}_{L}^{\gamma}, \\
& \mathbf{W}_{\mathrm{L}}^{(I I)}=\mathrm{L}_{\dot{a} b \dot{b}, \dot{d} d}^{(I I)}\left[\epsilon_{\alpha \beta \gamma} \mathbf{E}_{L \dot{a}} \mathbf{U}_{L \dot{b}}^{\alpha} \mathbf{U}_{L \dot{c}}^{\beta} \mathbf{D}_{L \dot{d}}^{\gamma}+\epsilon_{\alpha \beta \gamma} \nu_{L \dot{a}} \mathbf{D}_{L \dot{b}}^{\alpha} \mathbf{U}_{L \dot{c}}^{\beta} \mathbf{D}_{L \dot{d}}^{\gamma}\right], \\
& \mathrm{W}_{\mathrm{L}}^{(I I I)}=\mathrm{L}_{1 \dot{a} \hat{b}}^{(I I I)} \quad\left[\epsilon_{\alpha \beta \gamma} \mathbf{E}_{L \dot{a}} \mathbf{U}_{L \dot{b} \alpha}{ }^{\left(10_{16}\right)} \mathbf{t}_{L}^{\beta}{ }^{\left(10_{16}\right)} \mathbf{b}_{L}^{\gamma}+\epsilon_{\alpha \beta \gamma} \nu_{L \dot{a}} \mathbf{D}_{L \dot{b}}^{\left.\alpha{ }^{(10}{ }_{16}\right)} \mathbf{t}_{L}^{\beta}{ }^{\left(10_{16}\right)} \mathbf{b}_{L}^{\gamma}\right] \\
& +\mathrm{L}_{2}^{(I I I)}{ }_{a}^{(I I I)} \quad \epsilon_{\alpha \beta \gamma}{ }^{\left({ }_{16}\right)} \boldsymbol{\tau}_{L}{ }^{\left(10_{16}\right)} \mathbf{t}_{L}^{\alpha} \mathbf{U}_{L \dot{a}}^{\beta} \mathbf{D}_{L \dot{b}}^{\gamma} \\
& +\mathrm{L}_{2}^{(I I I)} \epsilon_{\alpha \beta \gamma}{ }^{\left(5_{16}\right)} \boldsymbol{\nu}_{L \tau}{ }^{\left(10_{16}\right)} \mathbf{b}_{L}^{\alpha} \mathbf{U}_{L \dot{a}}^{\beta} \mathbf{D}_{L \dot{b}}^{\gamma} .
\end{aligned}
$$

The coefficients L and R are defined in Tables 1 and 2. In computing them we have limited ourselves to one generation of $144+\overline{144}$ plet of Higgs. Thus the couplings with 120 plet mediation which are anti-symmetric in the generation indices vanish. 
Table 1 : Definition of parameters in Table 2

\begin{tabular}{|c|c|}
\hline A & $\frac{\cos ^{2} \vartheta_{\mathrm{T}}}{M_{\mathrm{T}_{2}}}+\frac{\sin ^{2} \vartheta_{\mathrm{T}}}{M_{\mathrm{T}_{3}}}$ \\
\hline B & $\frac{\sin ^{2} \vartheta_{\mathrm{T}}}{M_{\mathrm{T}_{2}}}+\frac{\cos ^{2} \vartheta_{\mathrm{T}}}{M_{\mathrm{T}_{3}}}$ \\
\hline $\mathrm{C}$ & $\left(\frac{1}{M_{\mathrm{T}_{2}}}-\frac{1}{M_{\mathrm{T}_{3}}}\right) \cos \vartheta_{\mathrm{T}} \sin \vartheta_{\mathrm{T}}$ \\
\hline $\mathrm{X}_{1 a ́ b}$ & $8 p\left(4 \xi_{a}^{(10)(+)}-\lambda_{a, \dot{b}}^{(45)}\right)$ \\
\hline $\mathrm{X}_{2 a ́ b}$ & $2 p\left(-16 \xi_{a}^{(10)(+)}-\lambda_{a, b}^{(45)}+5 \lambda_{a, b}^{(54)}\right)$ \\
\hline 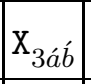 & $20 p\left(-4 \xi_{a}^{(10)(+)}+\lambda_{a, b}^{(54)}\right)$ \\
\hline $\mathrm{Y}_{1 a ́ b}$ & $32 q\left(\zeta_{a ́ b}^{(10)(+)}+\frac{2}{15} \varrho_{a ́ b}^{(126, \overline{126})(+)}\right)$ \\
\hline 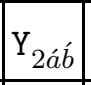 & $\frac{q}{\sqrt{5}}\left(16 \zeta_{a ́ b}^{(10)(+)}+2 \lambda_{a, b}^{(10)}-\frac{3}{5} \varrho_{a ́ b}^{(126, \bar{b}(\overline{126})(+)}\right)$ \\
\hline $\mathrm{Y}_{3 a ́ b}$ & $\frac{q}{\sqrt{5}}\left(-16 \zeta_{a ́ b}^{(10)(+)}+3 \lambda_{a, b}^{(10)}-\frac{14}{15} \varrho_{a ́ b}^{(126, \overline{126})(+)}\right)$ \\
\hline $\mathrm{Y}_{4 a ́}$ & $q\left(-80 \zeta_{a}^{(10)(+)}+\frac{3}{5} \varrho_{a ́ b}^{(126, \bar{b} \bar{b})(+)}\right)$ \\
\hline $\mathrm{Y}_{5 a ́ b}$ & $2 q\left(40 \zeta_{a}^{(10)(+)}-\frac{1}{5} \varrho_{a}^{(126, \bar{b} \overline{126})(+)}\right)$ \\
\hline
\end{tabular}

Table 2 : Coefficients of $L L L L$ and $R R R R$ baryon and lepton number violating dimension five operators

\begin{tabular}{|c|c|}
\hline $\mathrm{R}^{(I)}$ & $f_{33}^{(10)} f_{33}^{(45)} \sin \theta_{u \mathrm{~b}} \cos \theta_{u \mathrm{t}}\left[\left(-\frac{1}{5} \mathrm{~A}-\mathrm{B}+\frac{2}{\sqrt{5}} \mathrm{C}\right) \sin \theta_{u} \tau \cos \theta_{u \mathrm{t}}+\left(-\frac{1}{5} \mathrm{~A}+\mathrm{B}+\frac{1}{\sqrt{2}} \frac{1}{M_{\mathrm{T}_{4}}}\right) \cos \theta_{u} \tau \sin \theta_{u \mathrm{t}}\right]$ \\
\hline 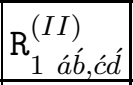 & 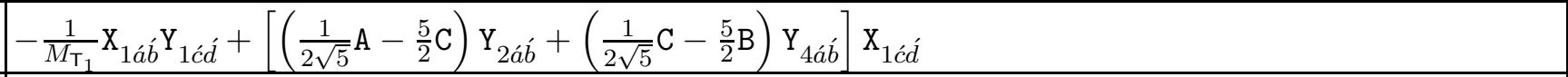 \\
\hline 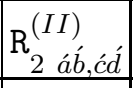 & $-\frac{128}{15} \frac{p q}{M_{\mathrm{T}_{4}}} \varrho_{a \dot{a} \hat{b}}^{(126 \overline{126})(+)} \lambda_{a, \hat{b}}^{(45)}$ \\
\hline $\mathrm{R}_{1}^{(I I I)}$ & $2 \sqrt{2} \frac{1}{M_{\mathrm{T}_{1}}} f_{33}^{(45)} \cos \theta_{u \mathrm{~b}} \sin \theta_{u \mathrm{t}} \mathrm{Y}_{1 a ́ b}-f_{33}^{(10)}\left(\frac{1}{20 \sqrt{2}} \mathrm{~A}+\frac{5}{4 \sqrt{2}} \mathrm{~B}-\frac{3}{2 \sqrt{10}} \mathrm{C}\right) \sin \theta_{u \mathrm{~b}} \cos \theta_{u \mathrm{t}} \mathrm{X}_{1 a ́ b}$ \\
\hline $\mathrm{R}_{2 a ́}^{(I I I)}$ & 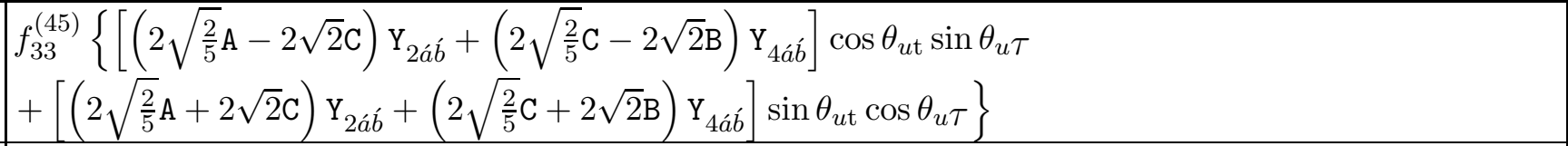 \\
\hline $\mathrm{R}_{3 a ́ b}^{(I I I)}$ & $-4 \sqrt{2} \frac{p}{M_{\mathrm{T}_{4}}} f_{33}^{(10)} \sin \theta_{u \mathrm{~b}} \cos \theta_{u \tau} \lambda_{a, \hat{b}}^{(45)}$ \\
\hline $\mathrm{R}_{4 \dot{a} \dot{b}}^{(I I I)}$ & $\frac{16}{15} \frac{q}{M_{\mathrm{T}_{4}}} f_{33}^{(45)} \cos \theta_{u \mathrm{~b}} \sin \theta_{u \tau} \varrho_{a ́ b}^{(126, \overline{126})(+)}$ \\
\hline $\mathrm{L}_{1}^{(I)}$ & $-f_{33}^{(10)} f_{33}^{(45)}\left(\frac{1}{5} \mathrm{~A}+\frac{1}{\sqrt{5}} \mathrm{C}\right) \sin \left(\theta_{v \mathrm{~b}}+\theta_{v \mathrm{t}}\right) \sin \theta_{v} \tau \cos \theta_{v \mathrm{t}}$ \\
\hline $\mathrm{L}_{2}^{(I)}$ & $-f_{33}^{(10)} f_{33}^{(45)}\left(\frac{1}{5} \mathrm{~A}+\frac{1}{\sqrt{5}} \mathrm{C}\right) \sin \left(\theta_{v \mathrm{~b}}+\theta_{v \mathrm{t}}\right) \sin \theta_{v \nu_{\tau}} \cos \theta_{v \mathrm{~b}}$ \\
\hline $\mathrm{L}_{a ́ b, c ́ d}^{(I I)}$ & 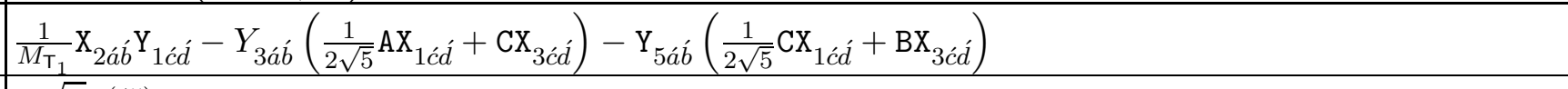 \\
\hline $\mathrm{L}_{1}^{(I I I)} \dot{a} \dot{b}$ & $2 \sqrt{\frac{2}{5}} f_{33}^{(45)}\left(\mathrm{AY}_{3 a ́ b}+\mathrm{CY}_{5 a ́ b}\right) \sin \left(\theta_{v \mathrm{~b}}+\theta_{v \mathrm{t}}\right)$ \\
\hline $\begin{array}{l}\mathrm{L}_{2}^{(I I I)} \\
2 a ́ b\end{array}$ & 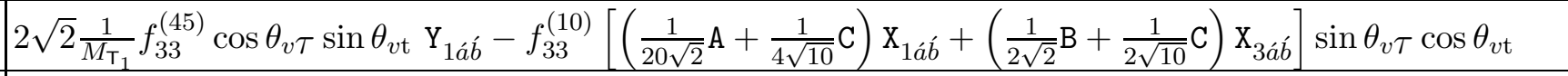 \\
\hline $\mathrm{L}_{3 a ́ b}^{(I I I)}$ & 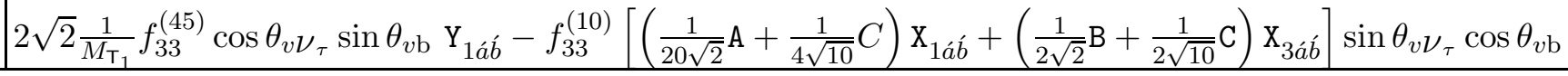 \\
\hline
\end{tabular}


[1] J. C. Pati and A. Salam, Phys. Rev. D 10, 275 (1974) [Erratum-ibid. D 11, 703 (1975)].

[2] H. Georgi and S. L. Glashow, Phys. Rev. Lett. 32, 438 (1974).

[3] H. Georgi, in Particles and Fields (edited by C.E. Carlson), A.I.P., 1975; H. Fritzch and P. Minkowski, Ann. Phys. 93(1975)193.

[4] P. Nath and P. F. Perez, Phys. Rept. 441, 191 (2007) arXiv:hep-ph/0601023.

[5] S. Dimopoulos and H. Georgi, Nucl. Phys. B 193, 150 (1981).

[6] A. H. Chamseddine, R. Arnowitt and P. Nath, Phys. Rev. Lett. 49, 970 (1982).

[7] S. Weinberg, Phys. Rev. D26, 287 (1982); N. Sakai and T. Yanagida, Nucl. Phys. B197, 533 (1982); S. Dimopoulos, S. Raby and F. Wilczek, Phys. Lett. B112, 133 (1982); J. Ellis, D.V. Nanopoulos and S. Rudaz, Nucl. Phys. B202, 43 (1982).

[8] P. Nath, A. H. Chamseddine and R. Arnowitt, Phys. Rev. D 32, 2348 (1985); Phys. Lett. B 156, 215 (1985); J. Hisano, H. Murayama and T. Yanagida, Nucl. Phys. B402, 46 (1993); T. Goto, T. Nihei and J. Arafune, Phys. Rev. D52, 505 (1995); K. S. Babu and S. M. Barr, Phys. Lett. B 381, 137 (1996); P. Nath and R. Arnowitt, Phys. Rev. D 38, 1479 (1988); T. Goto and T. Nihei, Phys. Rev. D59, 115009 (1999).

[9] H. Murayama and A. Pierce, Phys. Rev. D 65, 055009 (2002) arXiv:hep-ph/0108104.

[10] R. Dermisek, A. Mafi and S. Raby, Phys. Rev. D 63, 035001 (2001) arXiv:hep-ph/0007213.

[11] K. Kobayashi et al. [Super-Kamiokande Collaboration], Phys. Rev. D 72, 052007 (2005) arXiv:hep-ex/0502026.

[12] W. M. Yao et al. [Particle Data Group], J. Phys. G 33, 1 (2006).

[13] B. Bajc, P. Fileviez Perez and G. Senjanovic, Phys. Rev. D 66, 075005 (2002); D. EmmanuelCosta and S. Wiesenfeldt, Nucl. Phys. B 661, 62 (2003) arXiv:hep-ph/0302272].

[14] P. Nath, Phys. Lett. B 381, 147 (1996) arXiv:hep-ph/9602337); Phys. Rev. Lett. 76, 2218 (1996) arXiv:hep-ph/9512415.

[15] T. Ibrahim and P. Nath, Phys. Rev. D 62, 095001 (2000).

[16] K. S. Babu and S. M. Barr, Phys. Rev. D 48, 5354 (1993); Z. Chacko and R.N. Mohapatra, Phys.Rev. D59, 011702 (1999); Phys. Rev. Lett. 82, 2836 (1999); Z. Berezhiani, Z. Tavartkiladze and M. Vysotsky, hep-ph/9809301; I. Gogoladze and A. Kobakhidze, Phys. Atom. Nucl. 60 (1997) 126 [Yad. Fiz. 60N1 (1997) 136]; T. Dasgupta, P. Mamales and P. Nath, Phys. 
Rev. D 52, 5366 (1995) arXiv:hep-ph/9501325]; G. Altarelli, F. Feruglio, and I. Masina, JHEP 0011, 040 (2000); Q. Shafi and Z. Tavartkiladze, Phys. Lett. B487, 145 (2000); N. Maekawa, Prog. Theor. Phys. 106, 401 (2001); K. Turzynski, JHEP 0210 (2002) 044.

[17] B. Dutta, Y. Mimura and R. N. Mohapatra, Phys. Rev. Lett. 94, 091804 (2005) arXiv:hep-ph/0412105.

[18] T. Ibrahim and P. Nath, Phys. Rev. D 58, 111301 (1998); Phys. Rev. D 61, 093004 (2000) arXiv:hep-ph/9910553. For a review see, T. Ibrahim and P. Nath, "CP violation from standard model to strings," arXiv:0705.2008 [hep-ph].

[19] R. Arnowitt and P. Nath, Phys. Rev. D 49, 1479 (1994) arXiv:hep-ph/9309252.

[20] G. Segre and H. A. Weldon, Phys. Rev. Lett. 44, 1737 (1980); H. S. Tsao, Phys. Rev. D 24, 791 (1981); L. Arnellos and W. J. Marciano, Phys. Rev. Lett. 48, 1708 (1982); P. Eckert, J. M. Gerard, H. Ruegg and T. Schucker, Phys. Lett. B 125, 385 (1983); I. Dorsner and P. F. Perez, Phys. Lett. B 642, 248 (2006) arXiv:hep-ph/0606062]; P. F. Perez, arXiv:0705.3589 [hep-ph].

[21] N. Haba and T. Ota, arXiv:hep-ph/0608244.

[22] V. Lucas and S. Raby, Phys. Rev. D54, 2261 (1996); Phys. Rev. D55, 6986 (1997).

[23] K.S. Babu, J.C. Pati and F. Wilczek, Nucl. Phys. B566 33 (2000); Phys. Lett. B 423, 337 (1998).

[24] K. S. Babu, I. Gogoladze, P. Nath and R. M. Syed, Phys. Rev. D 72, 095011 (2005) [arXiv: hep-ph/0506312.

[25] K. S. Babu, I. Gogoladze, P. Nath and R. M. Syed, Phys. Rev. D 74, 075004 (2006) arXiv:hep-ph/0607244.

[26] R.N. Mohapatra and B. Sakita, Phys. Rev. D21, 1062 (1980).

[27] F. Wilczek and A. Zee, Phys. Rev. D25, 553 (1982).

[28] P. Nath and R. M. Syed, Phys. Lett. B 506, 68 (2001); Nucl. Phys. B 618, 138 (2001); Nucl. Phys. B 676, 64 (2004); R. M. Syed, arXiv: hep-ph/0411054 arXiv: hep-ph/0508153.

[29] P. Nath and R. M. Syed, JHEP 0602, 022 (2006).

[30] N. Arkani-Hamed and S. Dimopoulos, JHEP 0506, 073 (2005) arXiv:hep-th/0405159.

[31] B. Kors and P. Nath, Nucl. Phys. B 711, 112 (2005) arXiv:hep-th/0411201].

[32] K. Nakamura, Int. J. Mod. Phys. A 18 (2003) 4053; C. K. Jung, UNO," arXiv:hep-ex/0005046 M. V. Diwan et al., arXiv:hep-ex/0306053; A. de Bellefon et al, contribution to the CERN strategy committee, Orsay 30/01/06; L. Mosca, Nucl. Phys. Proc. Suppl. 138 (2005) 203; 
A. Rubbia, arXiv:hep-ph/0407297; D. B. Cline, arXiv:astro-ph/0506546; L. Oberaurer, http://nnn05.in2p3.fr; See also T. Marrodan Undagoitia et al, Phys. Rev. D72, 075014 (2005);

A. Bueno et al., JHEP 0704, 041 (2007) arXiv:hep-ph/0701101.

[33] O. Lebedev, H. P. Nilles, S. Raby, S. Ramos-Sanchez, M. Ratz, P. K. S. Vaudrevange and A. Wingerter, arXiv:0708.2691 [hep-th]. 